\title{
DECOMPOSABILITY OF HOMOTOPY LENS SPACES AND FREE CYCLIC GROUP ACTIONS ON HOMOTOPY SPHERES
}

\author{
BY
}

\section{KAI WANG}

ABSTRACT. Let $\rho$ be a linear $Z_{n}$ action on $\mathrm{c}^{m}$ and let $\rho$ also denote the induced $Z_{n}$ action on $S^{2 p-1} \times D^{2 q}, D^{2 p} \times S^{2 q-1}$ and $S^{2 p-1} \times s^{2 q-1}$ where $p=[m / 2]$ and $q=m-p$. A free differentiable $Z_{n}$ action $\left(\Sigma^{2 m-1}, \mu\right)$ on a homotopy sphere is $\rho$-decomposable if there is an equivariant diffeomorphism $\Phi$ of $\left(s^{2 p-1} \times s^{2 q-1}, \rho\right)$ such that $\left(\Sigma^{2 m-1}, \mu\right)$ is equivalent to $(\Sigma(\Phi)$, $A(\Phi))$ where $\Sigma(\Phi)=s^{2 p-1} \times D^{2 q} \cup_{\Phi} D^{2 p} \times s^{2 q-1}$ and $A(\Phi)$ is a uniquely determined action on $\Sigma(\Phi)$ such that $A(\Phi) \mid S^{2 p-1} \times D^{2 q}=\rho$ and $A(\Phi) \mid D^{2 p} \times$ $s^{2 q-1}=\rho$. A homotopy lens space is $\rho$-decomposable if it is the orbit space of a $\rho$-decomposable free $Z_{n}$ action on a homotopy sphere. In this paper, we will study the decomposabilities of homotopy lens spaces. We will also prove that for each lens space $L^{2 m-1}$, there exist infinitely many inequivalent free $z_{n}$ actions on $s^{2 m-1}$ such that the orbit spaces are simple homotopy equivalent to $L^{2 m-1}$.

0. Introduction. Let $A$ be the antipodal map and let $\Phi$ be an equivariant diffeomorphism of $\left(S^{p} \times S^{p}, A\right)$ where $A(x, y)=(-x,-y)$. Then there is a uniquely determined free involution $A(\Phi)$ on $\Sigma(\Phi)$ where

$$
\Sigma(\Phi)=S^{p} \times D^{p+1} \cup_{\Phi} D^{p+1} \times S^{p}
$$

such that the inclusions $S^{p} \times D^{p+1} \rightarrow \Sigma(\Phi), D^{p+1} \times S^{p} \rightarrow \Sigma(\Phi)$ are equivariant. In [9], Livesay and Thomas have proved the following:

THEOREM 0.1. Let $\left(\Sigma^{2 p+1}, T\right)$ be a free involution on a homotopy sphere $\Sigma^{2 p+1}$, then there is an equivariant diffeomorphism $\Phi$ of $\left(S^{p} \times S^{p}, A\right)$ such that $\left(\Sigma^{2 p+1}, T\right)$ is equivalent to $(\Sigma(\Phi), A(\Phi))$.

For $n \geqslant 2$, let $\rho$ be a linear $Z_{n}$ action on $\mathbf{C}^{m}$ which is free on $\mathbf{C}^{m}-0$. Let $p=[m / 2]$ and $q=m-p$. Then $S^{2 m-1}, S^{2 p-1} \times D^{2 q}, D^{2 p} \times S^{2 q-1}$ and $S^{2 p-1} \times S^{2 q-1}$ are invariant subspaces and the induced actions are free. We

Received by the editors January 27, 1975.

AMS (MOS) subject classifications (1970). Primary 57E15, 57E25, 57E30.

Key words and phrases. Wall groups, homotopy smoothings, normal invariants, decomposable homotopy lens spaces. 
will use the same notation $\rho$ to denote the induced actions. If $\Phi$ is an equivariant diffeomorphism of $\left(S^{2 p-1} \times S^{2 q-1}, \rho\right)$, then there is a uniquely determined free $Z_{n}$ action $A(\Phi)$ on $\Sigma(\Phi)$ where

$$
\Sigma(\Phi)=S^{2 p-1} \times D^{2 q} \cup_{\Phi} D^{2 p} \times S^{2 q-1} \text {. }
$$

such that the inclusions $S^{2 p-1} \times D^{2 q} \rightarrow \Sigma(\Phi)$ and $D^{2 p} \times S^{2 q-1} \rightarrow \Sigma(\Phi)$ are equivariant. In [9], using the same argument, they have also proved the following:

THEOREM 0.2. Let $\left(\Sigma^{4 m-1}, \mu\right)$ be a free $Z_{3}$ action on a homotopy sphere, then there is an equivariant diffeomorphism $\Phi$ of $\left(S^{2 m-1} \times S^{2 m-1}, \rho\right)$ such that $\left(\Sigma^{4 m-1}, \mu\right)$ is equivalent to $(\Sigma(\Phi), A(\Phi))$.

The proofs use the following well-known facts: (a) $J: K O\left(R P^{p}\right) \rightarrow J\left(R P^{p}\right)$ and $J: K O\left(L^{4 m-1}\left(Z_{3}\right)\right) \rightarrow J\left(L^{4 m-1}\left(Z_{3}\right)\right)$ are isomorphisms [1], and (b) $W h\left(Z_{2}\right)$ and $W h\left(Z_{3}\right)$ are zero [11]. The argument breaks down when $n \geqslant 4$. Hence, they asked if there are similar properties for $Z_{n}$ actions for $n \geqslant 4$ [10]. For convenience we say a free $Z_{n}$ action on a homotopy sphere is $\rho$-decomposable if it is equivalent to an action of the form $(\Sigma(\Phi), A(\Phi))$. For a more precise definition, see Definition 2.1. It turns out that the answer to their question is negative and there are necessary and sufficient conditions in order that a free $Z_{n}$ action on a homotopy sphere be $\rho$-decomposable. Since the orbit space $\Sigma^{2 m-1} / \mu$ of a free $Z_{n}$ action $\left(\Sigma^{2 m-1}, \mu\right)$ on a homotopy sphere is a homotopy lens space, each homotopy lens space is the orbit space of a free $Z_{n}$ action on a homotopy sphere and the equivalence class of $\left(\Sigma^{2 m-1}, \mu\right)$ up to automorphisms of $Z_{n}$ is uniquely determined by the diffeomorphism class of $\Sigma^{2 m-1} / \mu$. Thus the criterions on the decomposabilities can be stated in terms of orbit spaces. So we make the following definition. A homotopy lens space is $\rho$-decomposable if the corresponding action is $\rho$-decomposable. For a more precise definition, see Definition 2.2. The question of Livesay and Thomas is completely answered by the following:

THEOREM 0.3. A homotopy lens space $X^{2 m-1}$ is o-decomposable if and only if there is a simple homotopy equivalence $f: X^{2 m-1} \rightarrow L^{2 m-1}$ such that $\eta\left(\left[X^{2 m-1}, f\right]\right)\left|L^{2 p-1}=0, \eta\left(\left[X^{2 m-1}, f\right]\right)\right| L^{2 q-1}=0$, where $\eta$ is the normal map and $L^{2 p-1}=S^{2 p-1} / \rho, L^{2 q-1}=S^{2 q-1} / \rho$.

On the other hand, Milnor has constructed infinitely many inequivalent free $Z_{n}$ actions on $S^{2 m-1}$ such that the orbit spaces are not simple homotopy equivalent to any lens space [13]. However, by the Classification Theorem of Browder, Petrie and Wall [3], in each simple homotopy class of a lens space, there are infinitely many free $Z_{n}$ actions on homotopy spheres. Since the group of homotopy spheres is finite [7], one may conjecture that in each simple ho- 
motopy class of a lens space, there are infinitely many free $Z_{n}$ actions such that the underlying manifold is $S^{2 m-1}$. It turns out that a much stronger result is true. This is the following:

THEOREM 0.4. Let $\Sigma^{2 m-1}$ be a homotopy sphere which supports a free $Z_{n}$ action $\mu$ where $m \geqslant 3$. If $n \geqslant 3$ or $n=2$ and $m$ is even, then $\Sigma^{2 m-1}$ supports infinitely many inequivalent free $Z_{n}$ actions of which the orbit spaces are simple homotopy equivalent to $\Sigma^{2 m-1} / \mu$.

This paper is organized as follows. In $\S 1$, we will summarize the results of surgery theory which will be used in later sections. In $\$ 2$, we will prove Proposition 2.3 and Theorem 2.4 which imply Theorem 0.3. Most results in this section have been announced in [18]. In $\S 3$, we will prove Theorem 0.4 which is Theorem 3.1. It is understood that we are working in the differentiable category and $M \cong N$ means $M$ and $N$ are diffeomorphic.

1. Surgery on the compact manifolds. In this section we will recall the theory of surgery on the compact manifolds. The main reference will be Wall's book [17].

Let $X^{m}$ be a compact oriented manifold of dimension $m$ with boundary (possibly empty). We consider pairs $\left(M^{m}, f\right)$, where $M^{m}$ is a compact oriented manifold of the same dimension and $f:\left(M^{m}, \partial M^{m}\right) \rightarrow\left(X^{m}, \partial X^{m}\right)$ is a (simple) homotopy equivalence of pairs such that $f \mid \partial M^{m}$ is a diffeomorphism. $\left(M^{m}, f\right)$ is called a (simple) homotopy smoothing of $X^{m}$. Two pairs $\left(M_{1}^{m}, f_{1}\right)$ and $\left(M_{2}^{m}\right.$, $f_{2}$ ) are equivalent if there is a diffeomorphism $d: M_{1}^{m} \rightarrow M_{2}^{m}$ such that $f_{1}$ is homotopic to $f_{2} \cdot d$ relative to $\partial M_{1}^{m}$. Let $\left[M^{m}, f\right]$ be the equivalence class of $\left(M^{m}, f\right)$. For $\epsilon=h$ or $s$, let $S^{\epsilon}\left(X^{m}, \partial X^{m}\right)$ be the set of equivalence classes of (simple if $\epsilon=s$ ) homotopy smoothings of $X^{m}$.

Let $f:\left(M^{m}, \partial M^{m}\right) \rightarrow\left(X^{m}, \partial X^{m}\right)$ be a (simple) homotopy smoothing. Let $g$ be a homotopy inverse of $f$ such that $g \mid \partial X^{m}=\left(f \mid \partial M^{m}\right)^{-1}$. For $k$ sufficiently large, we may approximate $g \times 0: X^{m} \rightarrow M^{m} \times R^{k}$ by an embedding $\bar{g}$. Let $\nu$ be the normal bundle of the embedding $\bar{g} . \nu \mid \partial X^{m}$ is trivial and there is a canonical fiber homotopy trivialization for $\nu$. Let $G / O$ be the classifying space for fiber homotopy trivialization of vector bundles. Then $\left(M^{m}, f\right)$ determines an element $\eta\left(M^{m}, f\right)$ in $\left[X^{m} / \partial X^{m}, G / O\right] . \eta\left(M^{m}, f\right)$ depends only on the equivalence class $\left[M^{m}, f\right]$ and thus defines a map $\eta: S^{\epsilon}\left(X^{m}, \partial X^{m}\right) \rightarrow$ $\left[X^{m} / \partial X^{m}, G / O\right]$.

For each integer $k$, let $L_{k}^{\epsilon}\left(\pi_{1}\left(X^{m}\right)\right)$ be the Wall group of $\pi_{1}\left(X^{m}\right)$ [17]. For each $\xi \in\left[X^{m} / \partial X^{m}, G / O\right]$, there is a surgery obstruction $\sigma(\xi)$ in $L_{m}^{e}\left(\pi_{1}(X)\right)$ such that $\sigma(\xi)=0$ if and only if there is $\alpha \in S^{\epsilon}\left(X^{m}, \partial X^{m}\right)$ so that $\eta(\alpha)=\xi$ : Moreover, $L_{m+1}^{\epsilon}\left(\pi_{1}\left(X^{m}\right)\right)$ acts on $S^{\epsilon}\left(X^{m}, \partial X^{m}\right)$ such that for $\alpha, \beta \in S^{\epsilon}\left(X^{m}, \partial X^{m}\right)$ 
and $\eta(\alpha)=\eta(\beta)$, there is $x \in L_{m+1}^{\epsilon}\left(\pi_{1}\left(X^{m}\right)\right)$ such that $\beta=\omega(x, \alpha)$.

Thus we have the following exact sequence which includes much of the theory of surgery as developed by Milnor, Kervaire, Browder, Novikov, Sullivan and Wall [17],

$$
L_{m+1}^{\epsilon}\left(\pi_{1}\left(X^{m}\right)\right) \stackrel{\omega}{\rightarrow} S^{\epsilon}\left(X^{m}, \partial X^{m}\right) \stackrel{\eta}{\rightarrow}\left[X^{m} / \partial X^{m}, G / O\right] \stackrel{\sigma}{\rightarrow} L_{m}^{\epsilon}\left(\pi_{1}\left(X^{m}\right)\right) .
$$

Let $Y^{m} \subset$ int $X^{m}$ be a submanifold of same dimension. Suppose $g:\left(N^{m}\right.$, $\left.\partial N^{m}\right) \rightarrow\left(Y^{m}, \partial Y^{m}\right)$ is a (simple) homotopy smoothing. Let $M^{m}=\left(X^{m}-\right.$ int $\left.Y^{m}\right) \cup_{(g \mid \partial N)} N^{m}$ and define $f: M^{m} \rightarrow X^{m}$ by $f \mid X^{m}-$ int $Y=$ identity and $f \mid N^{m}=g$. It is easy to see that $\left(M^{m}, f\right)$ is a (simple) homotopy smoothing of $X^{m}$ and $\left[M^{m}, f\right]$ depends only on $\left[N^{m}, g\right]$. Thus we have a well-defined map a: $S^{\epsilon}\left(Y^{m}, \partial Y^{m}\right) \rightarrow S^{\epsilon}\left(X^{m}, \partial X^{m}\right)$. Note that $X^{m} /\left(X^{m}-\right.$ int $\left.Y^{m}\right)$ is homeomorphic to $Y^{m} / \partial Y^{m}$. The collapsing map $X^{m} / \partial X^{m} \rightarrow X^{m} /\left(X^{m}-\right.$ int $\left.Y^{m}\right)$ together with the homeomorphism induces a homomorphism $b:\left[Y^{m} / \partial Y^{m}, G / O\right]$ $\rightarrow\left[X^{m} / \partial X^{m}, G / O\right]$. Then we have the following theorem which is an easy consequence of the general theory.

THEOREM 1.1. Let $Y^{m} \subset$ int $X^{m}$ be a submanifold such that $\pi_{1}\left(Y^{m}\right) \approx$ $\pi_{1}\left(X^{m}\right) \approx \pi$. Then we have the following commutative diagram:

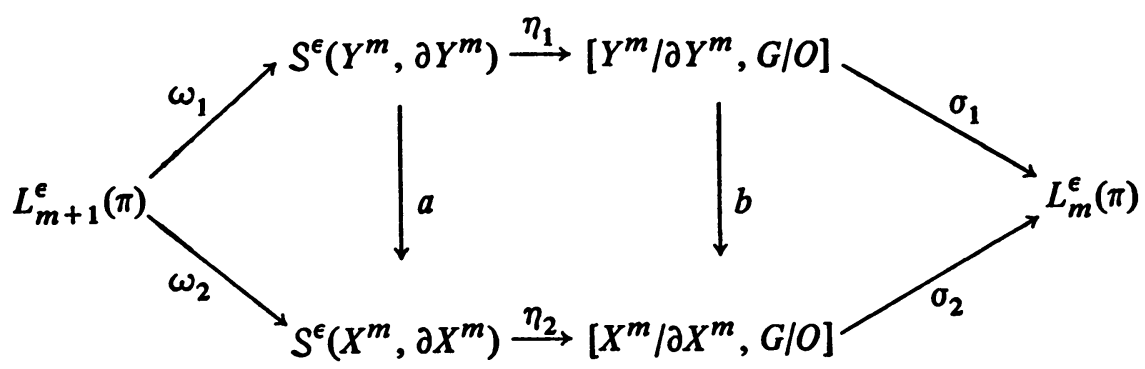

where $\epsilon=h$ or s.

Lemma 1.2. Let $\alpha \in S^{\epsilon}\left(X^{m}, \partial X^{m}\right)$. If $\eta_{2}(\alpha) \mid X^{m}-$ int $Y^{m}=0$, then there is $\gamma \in S^{\epsilon}\left(Y^{m}, \partial Y^{m}\right)$ such that $\alpha=a(\gamma)$.

Proof. Since $\eta_{2}(\alpha) \mid X^{m}-$ int $Y^{m}=0$, there is $\xi \in\left[Y^{m} / \partial Y^{m}, G / O\right]$ such that $\eta_{2}(\alpha)=b(\xi)$. But

$$
\sigma_{1}(\xi)=\sigma_{2} \cdot b(\xi)=\sigma_{2} \cdot \eta_{2}(\xi)=0 .
$$

Hence there is $\beta \in S^{\epsilon}\left(Y^{m}, \partial Y^{m}\right)$ such that $\eta_{1}(\beta)=\xi$. Since

$$
\eta_{2} \cdot a(\beta)=b \cdot \eta_{1}(\beta)=b(\xi)=\eta_{2}(\alpha),
$$

there is $x \in L_{m+1}^{e}(\pi)$ such that $\alpha=\omega_{2}(x, a(\beta))=a \cdot \omega_{1}(x, \beta)$. Let $\gamma=$ $\omega_{1}(x, \beta)$. Then we have $\alpha=a(\gamma)$. 
The following is a well-known result (see [17]). We shall omit the proof.

THEOREM 1.3. Let $K^{m-1}$ be a closed manifold. Then $S^{s}\left(K^{m-1} \times I, \partial\right)$ is an abelian group and $\omega: L_{m+1}^{s}\left(\pi_{1}\left(K^{m-1}\right)\right) \rightarrow S^{s}\left(K^{m-1} \times I, \partial\right)$ is a homomorphism.

The following useful result is due to Petrie [14].

THEOREM 1.4. Let $X^{2 m-1}$ be an oriented manifold such that $\pi_{1}\left(X^{2 m-1}\right)$ $=Z_{n}$. Then there is a subgroup $L_{2 m}^{\sim}\left(Z_{n}\right)$ of $L_{2 m}^{0}\left(Z_{n}\right)$ of rank $\gamma_{n, m}$ which acts freely on $S^{s}\left(X^{2 m-1}, \partial X^{2 m-1}\right)$, where

$$
\gamma_{n, m}= \begin{cases}{[n / 2]} & \text { if } m \text { is even, } \\ {[(n-1) / 2]} & \text { if } m \text { is odd. }\end{cases}
$$

Finally, let $f: M^{m} \rightarrow N^{m}$ be a homotopy equivalence. Then there is an element $\tau(f)$ in the Whitehead group $\operatorname{Wh}\left(\pi_{1}\left(N^{m}\right)\right)$ such that $\tau(f)=0$ if and only if $f$ is a simple homotopy equivalence [11]. The following lemma is a special case of the Sum Theorem of Whitehead torsions [8].

LEMMA 1.5. Let $Y^{m} \subset$ int $X^{m}$ be a submanifold such that $\pi_{1}\left(Y^{m}\right) \approx$ $\pi_{1}\left(X^{m}\right) \approx \pi$. Then we have the following commutative diagram

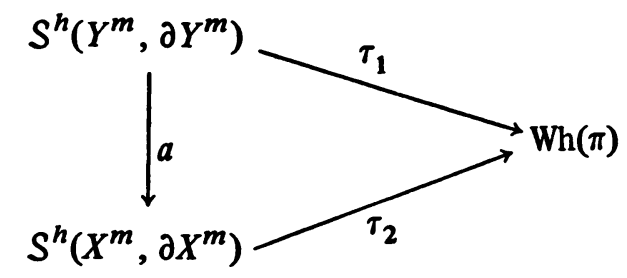

where $a$ is defined as in Theorem 1.1.

2. Decomposability of homotopy lens spaces. Let $\rho$ be a fixed linear action of $Z_{n}$ on $\mathbf{C}^{m}$ which is free on $\mathbf{C}^{m}-0$. Let $p=[m / 2]$ and $q=m-p$. Then $S^{2 m-1}, S^{2 p-1} \times D^{2 q}, D^{2 p} \times S^{2 q-1}$ and $S^{2 p-1} \times S^{2 q-1}$ are invariant subspaces. We will use the same notation $\rho$ to denote the induced actions. For an equivariant diffeomorphism $\Phi$ of $\left(S^{2 p-1} \times S^{2 q-1}, \rho\right)$, there is a well-defined $Z_{n}$ action $A(\Phi)$ on $\Sigma(\Phi)$ where

$$
\Sigma(\Phi)=S^{2 p-1} \times D^{2 q} \cup_{\Phi} D^{2 p} \times S^{2 q-1}
$$

such that the inclusions $S^{2 p-1} \times D^{2 q} \rightarrow \Sigma(\Phi)$ and $D^{2 p} \times S^{2 q-1} \rightarrow \Sigma(\Phi)$ are equivariant.

DEFINITION 2.1. A free $Z_{n}$ action $\left(\Sigma^{2 m-1}, \mu\right)$ on a homotopy sphere $\Sigma^{2 m-1}$ is $\rho$-decomposable if there is an equivariant diffeomorphism $\Phi$ of $\left(S^{2 p-1}\right.$ 
$\left.\times S^{2 q-1}, \rho\right)$ such that $\left(\Sigma^{2 m-1}, \mu\right)$ is equivalent to $(\Sigma(\Phi), A(\Phi))$.

It is well known that the orbit space of a free $Z_{n}$ action on a homotopy sphere is a homotopy lens space and every homotopy lens space is the orbit space of a free $Z_{n}$ action on a homotopy sphere. Thus the decomposition of a free $Z_{n}$ action on a homotopy sphere induces a decomposition of the orbit space which can be described as follows. Let

$$
\begin{gathered}
L^{2 m-1}=S^{2 m-1} / \rho, \quad M^{2 m-1}=\left(S^{2 p-1} \times D^{2 q}\right) / \rho, \\
N^{2 m-1}=\left(D^{2 p} \times S^{2 q-1}\right) / \rho
\end{gathered}
$$

and

$$
K^{2 m-2}=\left(S^{2 p-1} \times S^{2 q-1}\right) / \rho .
$$

DEFINITION 2.2. Let $X^{2 m-1}$ be a homotopy lens space. Then $X^{2 m-1}$ is said to be pre-p-decomposable if there is an h-cobordism $\left(W^{2 m-1} ; K^{2 m-2}\right.$, $K^{2 m-2}$ ) such that

$$
X^{2 m-1} \cong M^{2 m-1} \cup_{\text {id }} W^{2 m-1} \cup_{\text {id }} N^{2 m-1} .
$$

$X^{2 m-1}$ is said to be p-decomposable if $W^{2 m-1} \cong K^{2 m-2} \times I$.

It is easy to see that $X^{2 m-1}$ is $\rho$-decomposable if and only if there is a diffeomorphism $\varphi$ of $K^{2 m-2}$ such that $X^{2 m-1} \cong M^{2 m-1} \cup_{\varphi} N^{2 m-1}$. Hence a free $Z_{n}$ action on a homotopy sphere is $\rho$-decomposable if and only if its orbit space is $\rho$-decomposable. For convenience, we will study the decomposabilities of the homotopy lens spaces instead of the decomposabilities of the free $Z_{n}$ actions on homotopy spheres.

Let $L^{2 p-1}=\left(S^{2 p-1} \times 0\right) / \rho$ and $L^{2 q-1}=\left(0 \times S^{2 q-1}\right) / \rho$. Let $\eta: S^{\epsilon}\left(L^{2 m-1}\right)$ $\rightarrow\left[L^{2 m-1}, G / O\right]$ be the normal map. Then we have the following:

Proposition 2.3. Let $X^{2 m-1}$ be a homotopy lens space. Then $X^{2 m-1}$ is pre-p-decomposable if and only if there is a homotopy equivalence $f: X^{2 m-1}$ $\rightarrow L^{2 m-1}$ such that $\eta\left(\left[X^{2 m-1}, f\right]\right) \mid L^{2 p-1}=0$ and $\eta\left(\left[X^{2 m-1}, f\right]\right) \mid L^{2 q-1}=$ 0 .

Proof. Let $j: L^{2 p-1} \rightarrow L^{2 m-1}$ be the inclusion. Let $f: X^{2 m-1} \rightarrow$ $L^{2 m-1}$ be a homotopy equivalence and let $g_{1}$ be a homotopy inverse of $f$. Since $p=[m / 2], g_{1} j$ is homotopic to an embedding. By homotopy extension theorem, $g_{1}$ is homotopic to a homotopy equivalence $g$ such that $g j$ is an embedding. $g$ is also a homotopy equivalence of $f$. Let $\nu_{1}\left(\nu_{2}\right.$, respectively) be the normal bundle of $j$ (gj, respectively). Following Sullivan [16], for $k$ sufficiently large, we may approximate $g \times 0: L^{2 m-1} \rightarrow X^{2 m-1} \times R^{k}$ by an embedding $\bar{g}$ which extends gj. Let $\nu$ be the normal bundle of $\bar{g}$. It is clear that 
$(g) *\left(\nu_{2} \oplus \nu\right)$ is stably isomorphic to $j^{*} \nu_{1}$. By definition, $\eta\left(\left[X^{2 m-1}, f\right]\right)=$ $[\nu, t]$ where $t$ is a canonical fiber homotopy trivialization for $\nu$. Suppose that $\eta\left(\left[X^{2 m-1}, f\right]\right) \mid L^{2 p-1}=0$. Then $j^{*} \nu_{1}$ is stably isomorphic to $(g j)^{*} \nu_{2}$. Since $p=[m / 2], j^{*} \nu_{1}$ is isomorphic to $(g j)^{*} \nu_{2}$. It follows that $g \mid M^{2 m-1}$ is an embedding. Hence, if $\eta\left(\left[X^{2 m-1}, f\right]\right)\left|L^{2 p-1}=\eta\left(\left[X^{2 m-1}, f\right]\right)\right| L^{2 q-1}=0$, there is a homotopy equivalence $h: L^{2 m-1} \rightarrow X^{2 m-1}$ such that $h \mid M^{2 m-1}$ and $h \mid N^{2 m-1}$ are embeddings. Let

$$
W^{2 m-1}=X^{2 m-1}-\operatorname{int} h\left(M^{2 m-1}\right)-\operatorname{int} h\left(N^{2 m-1}\right) .
$$

It is routine to check that $W^{2 m-1}$ is an $h$-cobordism. Therefore $X^{2 m-1}$ is pre$\rho$-decomposable.

Conversely, suppose $X^{2 m-1}$ is pre- $\rho$-decomposable. Then there is an $h$ cobordism $\left(W^{2 m-1}, K^{2 m-2}, K^{2 m-2}\right)$ such that $X^{2 m-1} \cong M^{2 m-1} \cup_{\text {id }} W^{2 m-1}$ $\cup_{\text {id }} N^{2 m-1}$. Let $f: X^{2 m-1} \rightarrow L^{2 m-1}$ be a homotopy equivalence and let $g_{1}$ be a homotopy inverse of $f$. Let $k: L^{2 p-1} \rightarrow X^{2 m-1}$ be the inclusion. Let $\Sigma^{2 m-1}$ be the universal covering of $X^{2 m-1}$. Then $\Sigma^{2 m-1} \rightarrow X^{2 m-1}$ is a $(2 m-1)$-universal bundle for principal $Z_{n}$-bundles [15]. Both $k$ and $g_{1} j$ are classifying maps for $S^{2 p-1} \rightarrow L^{2 p-1}$, so $k$ and $g_{1} j$ are homotopic. By a theorem of Haefliger [5], $g_{1} j$ is isotopic to $k$. By the Isotopy Extension Theorem [12], $g_{1}$ is isotopic to a homotopy equivalence $g$ such that $g j=k$. $g$ is also a homotopy inverse of $f$. Again, for $k$ sufficiently large, we may approximate $g \times$ $0: L^{2 m-1} \rightarrow X^{2 m-1} \times R^{k}$ by an embedding $\bar{g}$ which extends $g \mid L^{2 p-1}$. Let $\nu$ be the normal bundle of $\bar{g}$. Then $\eta\left(\left[X^{2 m-1}, f\right]\right)=[\nu, t]$ where $t$ is a canonical fiber trivialization for $\nu$. Let $\nu_{1}\left(\nu_{2}\right.$, respectively) be the normal bundle of $j\left(k\right.$, respectively). Then $j^{*} \nu_{1}$ is isomorphic to $k^{*} \nu_{2}$ and there is a canonical fiber homotopy equivalence from $j^{*} v_{1}$ to $k^{*} v_{2}$ by a result of Hirsch [6]. It follows that $\eta\left(\left[X^{2 p-1}, f\right]\right)\left|L^{2 p-1}=[\nu, t]\right| L^{2 p-1}=0$. Similarly, we can prove $\eta\left(\left[X^{2 m-1}, f\right]\right) \mid L^{2 q-1}=0$.

THEOREM 2.4. Let $f: X^{2 m-1} \rightarrow L^{2 m-1}$ be a homotopy equivalence such that $\eta\left(\left[X^{2 m-1}, f\right]\right) \mid L^{2 p-1}=0$ and $\eta\left(\left[X^{2 m-1}, f\right]\right) \mid L^{2 q-1}=0$. Then $X^{2 m-1}$ is $\rho$-decomposable if and only if $f$ is a simple homotopy equivalence.

Proof. It is obvious that there is a diffeomorphism

$$
k: L^{2 m-1} \rightarrow M^{2 m-1} \cup_{\mathrm{id}} K^{2 m-2} \times I \cup_{\mathrm{id}} N^{2 m-1}
$$

such that $k\left(L^{2 p-1}\right)=L^{2 p-1}\left(k\left(L^{2 q-1}\right)=L^{2 q-1}, k\left(K^{2 m-2}\right)=K^{2 m-2} \times 1 / 2\right.$, respectively) and $k \mid L^{2 p-1}\left(L^{2 q-1}, K^{2 m-2}\right.$, respectively) is the identity. Hence, without loss of generality, we may assume

$$
L^{2 m-1}=M^{2 m-1} \cup_{\mathrm{id}} K^{2 m-2} \times I \cup_{\mathrm{id}} N^{2 m-1} .
$$


Since $L^{2 p-1}\left(L^{2 q-1}\right.$, respectively) is a deformation retract of $M^{2 m-1}\left(N^{2 m-1}\right.$, respectively), it follows from the assumption $\eta\left(\left[X^{2 m-1}, f\right]\right) \mid L^{2 p-1}=0$ and $\eta\left(\left[X^{2 m-1}, f\right]\right) \mid L^{2 q-1}=0$ that $\eta\left(\left[X^{2 m-1}, f\right]\right) \mid M^{2 m-1}=0$ and $\eta\left(\left[X^{2 m-1}, f\right]\right) \mid N^{2 m-1}=0$. Thus

$$
\eta\left(\left[X^{2 m-1}, f\right]\right) \mid X^{2 m-1}-\operatorname{int}\left(K^{2 m-2} \times I\right)=0 .
$$

Let

$$
a: S^{h}\left(K^{2 m-2} \times I, \partial\left(K^{2 m-2} \times I\right)\right) \rightarrow S^{h}\left(L^{2 m-1}\right)
$$

be the map defined as in Theorem 1.1. By Lemma 1.2, there is a homotopy smoothing $g: W^{2 m-1} \rightarrow K^{2 m-2} \times I$ such that $a\left(\left[W^{2 m-1}, g\right]\right)=\left[X^{2 m-1}, f\right]$. Since by Lemma $1.5, \tau_{2}\left(\left[X^{2 m-1}, f\right]\right)=\tau_{1}\left(\left[W^{2 m-1}, g\right]\right), f$ is a simple homotopy equivalence if and only if $g$ is a simple homotopy equivalence. It is also easy to show that $g$ is a simple homotopy equivalence if and only if $W^{2 m-1} \cong$ $K^{2 m-2} \times I$. Now the theorem is clear.

COROLlaRY 2.5. For $m \geqslant 3, n \geqslant 4$, there exist infinitely many distinct homotopy lens spaces simple homotopy equivalent to $L^{2 m-1}$ which are not preo-decomposable.

Proof. Consider the following exact sequence

$$
L_{2 m}^{0}\left(Z_{n}\right) \stackrel{\omega}{\rightarrow} S^{S}\left(L^{2 m-1}\right) \stackrel{\eta}{\rightarrow}\left[L^{2 m-1}, G / O\right] \stackrel{\sigma}{\rightarrow} L_{2 m-1}\left(Z_{n}\right)
$$

It has been proved by Browder [2] that $\eta$ is onto if $n$ is odd and according to Wall [17], the coker $\eta$ is $Z_{2}$ if $n$ is even. It is easy to see that for $n \geqslant 4$, there is $\xi \in \operatorname{Im} \eta$ such that $\xi \mid L^{2 p-1} \neq 0$. Suppose $\xi=\eta\left(\left[X^{2 m-1}, f\right]\right)$, then $X^{2 m-1}$ is not pre- $\rho$-decomposable. Furthermore, for $x \in L_{2 m}\left(Z_{n}\right)$,

$$
\eta\left(\omega\left(x,\left[X^{2 m-1}, f\right]\right)\right)\left|L^{2 p-1}=\eta\left(\left[X^{2 m-1}, f\right]\right)\right| L^{2 p-1} \neq 0 .
$$

Now the corollary follows easily from Theorem 1.4.

Corollary 2.6. For $m \geqslant 3$ and $n \neq 2,3,4,6$, there exist infinitely many distinct pre-p-decomposable lens spaces which are not $\rho$-decomposable.

Proof. For $u \in 2 \mathrm{Wh}\left(Z_{n}\right)$, there is an $h$-cobordism $\left(W_{u}^{2 m-1} ; K^{2 m-2}\right.$, $\left.K^{2 m-2}\right)$ such that $\tau\left(W_{u}^{2 m-1}, K^{2 m-2}\right)=u$. Let $X_{u}^{2 m-1}=M^{2 m-1} \cup_{\mathrm{id}} W_{u}^{2 m-1}$ $\cup_{\text {id }} N^{2 m-1}$. Let $\widetilde{W}_{u}^{2 m-1}\left(\widetilde{X}^{2 m-1}\right.$, respectively) be the universal covering of $W_{u}^{2 m-1}\left(X_{u}^{2 m-1}\right.$, respectively). Since $S^{2 p-1} \times S^{2 q-1}$ is simply-connected and $\widetilde{W}_{u}^{2 m-1} \cong S^{2 p-1} \times S^{2 q-1} \times I, \widetilde{X}_{u}^{2 m-1}$ is a homotopy sphere which supports a free $Z_{n}$ action such that the orbit space is $X_{u}^{2 m-1}$. Thus $X_{u}^{2 m-1}$ is a homotopy lens space which is clearly pre-p-decomposable. It is also clear that for $u \neq v$, $X_{u}^{2 m-1} \not X_{v}^{2 m-1}$. Now the corollary following the fact that $\operatorname{rank} \operatorname{Wh}\left(Z_{n}\right) \geqslant 1$ 
if $n \neq 2,3,4,6$, and $X_{u}^{2 m-1}$ is not simple homotopy equivalent to $L^{2 m-1}$.

COROllary 2.7. Let $m \geqslant 3$. For $n \geqslant 3$ or $n=2, m$ is even, there exist infinitely many distinct $p$-decomposable homotopy lens spaces.

Proof. Let $\omega: L_{2 m}^{0}\left(Z_{n}\right) \rightarrow S^{S}\left(L^{2 m-1}\right)$ be the action of $L_{2 m}^{0}\left(Z_{n}\right)$ on $S^{s}\left(L^{2 m-1}\right)$. It follows easily from Theorem 2.4 that if $X^{2 m-1}$ is a $\rho$-decomposable homotopy lens space then each homotopy lens space in the orbit of $\left[X^{2 m-1}, f\right]$ where $f$ is a suitable simple homotopy equivalence, is decomposable. $L^{2 m-1}$ is obviously $\rho$-decomposable. Now the corollary follows from Theorem 1.4 .

REMARK 2.8. We have studied the decomposable free $S^{1}$ actions on homotopy spheres in [19], [20].

REMARK 2.9. Theorem 2.4 and Corollary 2.6 have also been obtained independently by C. C. Liang.

3. Free $Z_{n}$ actions on homotopy spheres. Let $\left(\Sigma^{2 m-1}, \mu\right)$ be a free $Z_{n}$ action on a homotopy sphere $\Sigma^{2 m-1}$. Then it is well known that there is a lens space $L^{2 m-1}$ such that $\Sigma^{2 m-1} / \mu$ is homotopy equivalent to $L^{2 m-1}$. Let $X^{2 m-1}$ $=\Sigma^{2 m-1} / \mu$ and let $f: L^{2 m-1} \rightarrow X^{2 m-1}$ be a homotopy equivalence. $f \mid L^{2 p-1}$ is homotopic to an embedding. Let $P^{2 m-1}$ be the tubular neighborhood of this embedding and let $Q^{2 m-1}=X^{2 m-1}-$ int $P^{2 m-1}$. Let $\pi: \Sigma^{2 m-1} \rightarrow X^{2 m-1}$ be the projection. Then it is easy to see that $\pi^{-1}\left(P^{2 m-1}\right) \cong S^{2 p-1} \times D^{2 q}$. By an easy application of the $h$-cobordism theorem, we can show that $\pi^{-1}\left(Q^{2 m-1}\right)$ $\cong D^{2 p} \times S^{2 q-1}$. Let $\mu_{P}\left(\mu_{Q}\right.$, respectively) be the induced action on $S^{2 p-1} \times$ $D^{2 q}\left(D^{2 p} \times S^{2 q-1}\right.$, respectively). Note that in general, $\mu_{P}$ and $\mu_{Q}$ are not linear. For an equivariant diffeomorphism $\psi:\left(S^{2 p-1} \times S^{2 q-1}, \mu_{P}\right) \rightarrow\left(S^{2 p-1} \times\right.$ $\left.S^{2 q-1}, \mu_{Q}\right)$, let $B(\psi)$ be the uniquely determined $Z_{n}$ action on $\Sigma(\psi)$ such that the inclusions $\left(S^{2 p-1} \times D^{2 q}, \mu_{p}\right) \rightarrow(\Sigma(\psi), B(\psi))$ and $\left(D^{2 p} \times S^{2 q-1}, \mu_{Q}\right) \rightarrow$ $(\Sigma(\psi), B(\psi))$ are equivariant. It is clear that there is an equivariant diffeomorphism $\Phi:\left(S^{2 p-1} \times S^{2 q-1}, \mu_{P}\right) \rightarrow\left(S^{2 p-1} \times S^{2 q-1}, \mu_{Q}\right)$ such that $\left(\Sigma^{2 m-1}, \mu\right)$ is equivalent to $(\Sigma(\Phi) B(\Phi))$. Let $R^{2 m-2}=\partial\left(P^{2 m-1}\right)$. It is obvious that

$$
X^{2 m-1} \cong P^{2 m-1} \cup_{\mathrm{id}} R^{2 m-2} \times I \cup_{\mathrm{id}} Q^{2 m-1} .
$$

Consider the following commutative diagram:

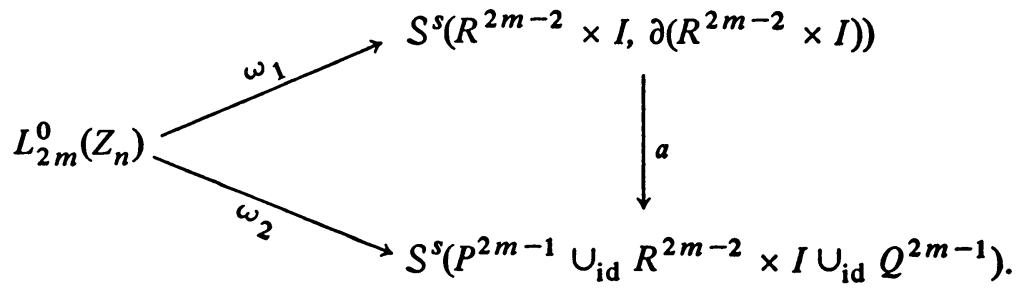


By Theorem 1.4, for $n \geqslant 3$ or $n=2$ and $m$ is even, there is an infinite subgroup $L_{2 m}^{\sim}\left(Z_{n}\right)$ of $L_{2 m}^{0}\left(Z_{n}\right)$ which acts freely on $S^{s}\left(P^{2 m-1} \cup_{\mathrm{id}} R^{2 m-2} \times I \cup_{\mathrm{id}}\right.$ $\left.Q^{2 m-1}\right)$. Let $x \in L_{2 m}^{\sim}\left(Z_{n}\right)$ be an element of infinite order. For each integer $k$, let $\omega_{2}(k x)=\left[X_{k}^{2 m-1}, f_{k}\right]$. Note that $X_{k}^{2 m-1}$ are distinct and are simple homotopy equivalent to $X^{2 m-1}$. It is easy to see that for each integer $k$, there is a representative $\left[R^{2 m-2} \times I, g_{k}\right]$ in $\omega_{1}(k x)$ such that $g_{k} \mid R^{2 m-2} \times 0=$ identity. Let $\Psi=g_{1} \mid R^{2 m-2} \times 1$. Since $\omega_{1}$ is a homomorphism, $g_{k} \mid R^{2 m-2} \times 1=\Psi^{k}$. It follows from $\omega_{2}=a \cdot \omega_{1}$ that $X_{k}^{2 m-1} \cong P^{2 m-1} \cup_{\Psi k} Q^{2 m-1}$. Let $\psi$ be the equivariant diffeomorphism of $\left(S^{2 p-1} \times S^{2 q-1}, \mu_{P}\right)$ which covers $\Psi$. Let $\Sigma_{k}^{2 m-1}$ be the homotopy sphere which supports a free $Z_{n}$ action such that its orbit space is $X_{k}^{2 m-1}$. It is clear that $\Sigma_{k}^{2 m-1} \cong \Sigma\left(\Phi \cdot \psi^{k}\right)$. By a lemma of [19], we can show that there is an infinite subset $\Lambda$ of integers such that $\Sigma_{k}^{2 m-1}$ is diffeomorphic to $\Sigma^{2 m-1}\left(=\Sigma_{0}^{2 m-1}\right)$ for $k \in \Lambda$. Thus we have proved the following:

THEOREM 3.1. Let $\Sigma^{2 m-1}$ be a homotopy sphere which supports a free $Z_{n}$ action $\mu$ where $m \geqslant 3$. If $n \geqslant 3$ or $n=2$ and $m$ is even, then $\Sigma^{2 m-1}$ supports infinitely many inequivalent free $Z_{n}$ actions of which the orbit spaces are simple homotopy equivalent to $\Sigma^{2 m-1} / \mu$.

In particular, we have

COROLlary 3.2. For each lens space $L^{2 m-1}, n \geqslant 3$, there exist infinitely many inequivalent free $Z_{n}$ actions on $S^{2 m-1}$ such that the orbit spaces are simple homotopy equivalent to $L^{2 m-1}$ if $n \geqslant 3$ or $n=2$ and $m$ is even.

REMARK 3.3. For $n \neq 2,3,4,6$, Milnor has shown that there exist infinitely many inequivalent free $Z_{n}$ actions on $S^{2 m-1}$ such that none of the orbit spaces is simple homotopy equivalent to a lens space [13].

REMARK 3.4. A weaker version of Theorem 3.1 has been announced in [18].

REMARK 3.5. A weaker version of the $S^{1}$ analogs of Theorem 3.1 has been proved in [19].

REMARK 3.6. The $S^{1}$-analogs of Theorem 3.1 have been proved by Burghelea [4] and which can also be proved by this method.

\section{REFERENCES}

1. J. F. Adams, On the groups $J(X)$. II, Topology 3 (1965), 137-171. MR 33 \#6626.

2. W. Browder, Free $Z_{p}$-actions on homotopy spheres, Topology of Manifolds (Proc. Inst., Univ. of Georgia, Athens, Ga., 1969), Markham, Chicago, Ill., 1970, pp. 217-226. MR 43 \#2720.

3. W. Browder, T. Petrie and C. T. C. Wall, The classification of free actions of cyclic 
groups of odd order on homotopy spheres, Bull. Amer. Math. Soc. 77 (1971), 455-459. MR 43 \#5547.

4. D. Burghelea, Free differentiable $S^{1}$ and $S^{3}$ actions on homotopy spheres, Ann. Sci. École Norm. Sup. (4) 5 (1972), 183-215. MR 46 \#6380.

5. A. Haefliger, Plongements différentiables des variétés dans variétés, Comment. Math. Helv. 36 (1961), 47-82. MR 26 \#3069.

6. M. Hirsch, On the fibre homotopy type of normal bundles, Michigan Math. J. 12 (1965), 225-229. MR 32 \#1724.

7. M. Kervaire and J. Milnor, Groups of homotopy spheres. I, Ann. of Math. (2) 77 (1963), 504-537. MR 26 \#5584.

8. K. W. Kwun and R. Szczarba, Product and sum theorems for Whitehead torsions, Ann. of Math. (2) 82 (1965), 183-190. MR 32 \#454.

9. G. R. Livesay and C. B. Thomas, Free $Z_{2}$ and $Z_{3}$ actions on homotopy spheres, Topology 7 (1968), 11-14. MR 36 \#3343.

10. Problem 9, Problems (Conf. on Transformation Groups, New Orleans, 1967), Springer-Verlag, Berlin and New York, 1968, pp. 235-236. 33 \#4922.

11. J. Milnor, Whitehead torsion, Bull. Amer. Math. Soc. 72 (1966), 358-426. MR

12. Some free actions of cyclic groups on spheres, Differential Analysis, Bombay Colloq., 1964, Oxford Univ. Press, London, 1964, pp. 37-42. MR 32 \#6482.

13. Lectures on the h-cobordism theorem, Princeton Univ. Press, Princeton,

N. J., 1965. MR 32 \#335.

14. T. Petrie, The Atiyah-Singer invariant, the Wall group and the function

$\left(t e^{x}+1\right) /\left(t e^{x}-1\right)$, Ann. of Math. (2) 92 (1974), 174-187.

15. N. E. Steenrod, Topology of flbre bundles, Princeton Math. Series, vol. 14, Princeton Univ. Press, Princeton, N. J., 1951. MR 12, 522.

16. D. Sullivan, Triangulating homotopy equivalence, Thesis, Princeton University, Princeton, N. J., 1965.

17. C. T. C. Wall, Surgery on compact manifolds, Academic Press, New York, 1970.

18. K. Wang, Decomposabilities of homotopy lens spaces, Bull. Amer. Math. Soc. 79 (1973), 389-392.

19. Differentiable $S^{1}$ actions on homotopy spheres, Invent. Math. 24 (1974), 51-63. MR 50 \#5830.

20. - Free $S^{1}$ actions and the group of diffeomorphisms, Trans. Amer. Math. Soc. 191 (1974), 113-127. MR 50 \#8577.

DEPARTMENT OF MATHEMATICS, STATE UNIVERSITY OF NEW YORK AT BUFFALO, AMHERST, NEW YORK 14226 